\title{
Fault section identification and location on a distribution feeder using travelling waves
}

\begin{abstract}
This paper describes how the fault generated travelling waves detected in the current signals at a single location on a distribution feeder can be used for fault section identification and location. The method identifies the fault section and the probable location of the fault by comparing the relative distance of each "peak" in the high frequency current signals to the known reflection points in the distribution feeder. The probable fault location is then used within a transient power system simulator that models the actual network. The resulting simulated current waveforms are then cross-correlated against the signal captured on the real network. If the estimated fault location is correct, the high frequency signatures in the simulated waveform will be similar to that of the measured waveforms and the crosscorrelation value will be a high positive value. Simulation studies using PSCAD/EMTDC and analysis using cross-correlation technique suggest that the method described can accurately locate a fault on a distribution feeder using measurement at a single location.
\end{abstract}

Keyword: Cross-correlation; Fault location; Time Trees; Travelling waves 